\title{
TCP muscle tensors: Theoretical analysis and potential applications in aerial robotic systems
}

\author{
A.E. Gomez-Tamm ${ }^{1}$, P. Ramon-Soria ${ }^{1}$, B.C. Arrue ${ }^{1}$, and A. Ollero ${ }^{1}$ \\ Group of Robotics Vision and Control. University of Seville, \\ agtamm@us.es, prs@us.es, barrue@us.es, aollero@us.es
}

\begin{abstract}
The use of aerial systems in a variety of real applications is increasing nowadays. These offer solutions to existing problems in ways that have never seen before thanks to their capability to perform perching, grasping or manipulating in inaccessible or dangerous places. Many of these applications require small-sized robots that can maneuver in narrow environments. However, these are required to have also strength enough to perform the desired tasks. This balance is sometimes unreachable due to the fact that traditional servomotors are too heavyweight for being carried by such small unmanned aerial systems (UAS). This paper, offers a innovative solution based on twisted and coiled polymers (TCP) muscles. These tensors have a high weight/strength ratio (up to 200 times) compared with traditional servos. In this work, the practical and modeling work done by the authors is presented. Then, a preliminary design of a bio-inspired claw for an unmanned aerial system (UAS) is shown. This claw has been developed using additive manufacturing techniques with different materials. Actuated with TCP, it is intrinsically compliant and offers a great force/weight ratio.
\end{abstract}

Keywords: soft robotics, bio-inspired robots, aerial robots, aerial manipulation

\section{Introduction}

The advances in unmanned aerial vehicles (UAV) over the years have promoted their use in manifold applications. However, there are still several limitations while working with these robots. These limitations affect mainly to three parameters: endurance, payload, and maneuverability. The balance between these parameters is essential during the design and development of UAV.

Due to that fact, researchers have centered their efforts in reducing the weight of these systems so that endurance and maneuverability could be optimized.

Our research, focuses on a particular type of UAS called Aerial Manipulators (AM). These aerial robots are equipped with limbs to extend their operation range. However, this equipment inevitably adds weight, and unbalances the robot.

Nowadays, the most used solution for actuating arms and legs are servo motors. The problem with this kind of devices is their weight. Authors in [16] 
developed a pair of novel lightweight arms with a total weight of $1.3 \mathrm{~kg}$ approximately. This design was extremely optimized, but it has a lower bound that cannot be overstepped due to the servos. Analyzing reviews in this matter [12, 7], it is easy to realize that most of the approaches center their efforts in optimizing other parameters of the manipulators. Some approaches explore different options such as using additive manufacturing techniques [2]. However, the use of this technology has a consequent trade-off between weight and robustness.

Authors in [5] developed lightweight arms based in one conventional servo per arm, but using tensors to reduce the moments in the joints. The problem with this approach is that it has intrinsic limitations. The maximum weight to lift and the working volume is reduced due to the fact that all the movements are dependent on the same servo.

Some projects like [10] have centered efforts in developing optimized aerial manipulators. Others like [3] have tried to develop soft actuators for industrial inspection. However, drastically reducing weight while adding softness to manipulators are still unresolved challenges.

Replacing servos by twisted and coiled polymers (TCP) muscles fibers [8] could be one all-time solution for reducing the total weight of AM. Moreover, these actuators have some desirable properties that may be of interest in aerial manipulation. One of them is that these are intrinsically compliant and add softness to the system. Recent research [4] has proved that adding compliance to the manipulator, reduce the perturbations on the aerial platform and improves the maneuverability. However, this has only been achieved until now, by adding springs or elastic bands to conventional servos. Authors in [15] developed a compliant lightweight manipulator for aerial robots, but the weight is still limited by the use of servo motors.

TCP muscles are however still an immature technology. There is a strong need to settle the pillars to help new researchers to work with them. TCP works thanks to nylon elasto-plastic behavior [6]. One principal characteristic is their shape memory property, the ability of undergoing deformation at one temperature and then recover its original length.

One of the limitations of using these muscles nowadays is the lack of manufacturers. These, are currently produced in laboratories with handcrafted techniques.

This manufacturing process has been described in various articles $[8,18]$. In this article, we briefly cover these steps to settle a general manufacturing process. Two different types of Nylon threads are normally used. The first one, used since the first paper published in 2014 [8], is a common fishing line thread that can be found in different diameters. The more diameter, the more weight it can lift. These wires are strong and resistant but are difficult to heat, even more, when embedded on aerial systems. In the first years, these were heated using hot water or air. However, these methods are quite inefficient and impracticable when working with UAV.

To overcome this issue, authors in [14] started using copper wires twisted over the nylon threads, so that the threads could be electrically heated. Authors 
in [20], on the other hand, used silver covered nylon thread, thought for clothes manufacturing, to obtain better performances in terms of heating transmission. Even though this was a great step into this research, problems appeared due to the fact that these wires are not designed to lift weight, leading to unexpected breakages. They also had much less diameter than the normal fishing line wires, limiting the force they can perform.

In this paper, the combination of both types of threads is presented. This is a novel combination idea. The purpose is to extract the best properties of each thread to obtain the best possible muscle fiber.

Finally, it is worth mentioning that the work on this TCP done for these last 5 years have centered their efforts in developing models of the muscles behavior [1]. For proving their utility, several robotic arms have been developed [5, 20, 13], and also hybrid small robots like [19]. Despite this, all the models mentioned do not bring in a real application for them, offering only functional proof of concept. In this work, the conception will be different, centering the effort in developing a system for a real application in the field of aerial robotics. This has not been explored yet and could have huge potential. Particularly, the GRIFFIN project aims to develop multi-function ornithopters with manipulation capabilities, that can perform various tasks. This kind of AM have stricter payload limitations, making vital the research of new forms of actuation. This paper is a first step in making those system capable of performing perching and grasping in a bioinspired compliant way and with much less payload requirements.

Following this idea, bio-inspired designs or the idea of developing systems based on the mother nature living beings is something researchers have tried to achieve in very different fields over the years. Some authors $[9,11]$ inspired their design in insects. Nevertheless, they still use classic servo-actuators, and only the mechanical design is bio-inspired. Other approaches such as [17] try to solve that issue, developing passive actuators. However, this solution presents limited actuation capabilities. The goal of this work is trying to develop an innovative solution that could offer a bio-inspired design. That is, emulating the births muscle capabilities using tensors and mimicking biological muscle configuration for enhance performance. This innovative research could lead to replace servomotors in aerial systems, getting closer to a biological behavior, and optimizing their payload.

The remainder of the article is organized as follow. Section 2 introduces the developed model to better understand the behaviour of TCP muscle fibers. Section 3 focus on the novel TCP muscle configuration and potential. Section 4 shows the application proposed for aerial manipulators, an ultra light weight bio-inspired claw. At last, Section 5 present the conclusions and the future lines of work.

\section{Muscle Models}

This section introduces the models developed to better understand the behavior of TCP muscle fibers. At first, the muscle manufacturing process is introduced. 
After that, the system is modeled using different test-bench configurations. At the end, the model conclusion is presented.

\section{$2.1 \quad$ Muscles behavior}

As mentioned in previous works such as [8], to grant that the manufacturing of the muscles is successful, it is required to have many details into consideration. This process is divided into the following steps: 1) Twisting; 2) Coiling; 3) Loading and 4) Annealing.

The process starts by spinning the nylon in the longitudinal direction. This is called twisting phase. The nylon starts to twist and generates an internal deformation. When the internal structure can not hold more torsion it reaches a collapse point, when the coiling starts. During the coiling, the nylon forms loops, or coils, over itself. Equation 1 is used to quantify the relation between the number of coils and weight for the coiling process according to the parameters of the fiber:

$$
\left\{\begin{array}{l}
N_{\text {coils }}=l_{\text {thread }} / \pi\left(d_{\text {rod }}+d_{\text {nylon }}\right) \\
m=\left(\pi\left(d_{\text {nylon }} / 2\right)^{2} T\right) / g
\end{array}\right.
$$

Where $N_{\text {coils }}$ means the number of coils of the finished muscle fiber, $d_{\text {rod }}$ is the diameter of the rod used for the coiling, if no rod is used (in the case of a self-coiled muscle fiber) this parameter is equal to zero, $d_{\text {nylon }}$ is the diameter of the nylon thread, $l_{\text {thread }}$ is the length of the nylon thread just before it starts to form the loops. $m$ is the weight used for the coiling, $T$ is the recommended load tension during coiling out of [8], and $g$ is the gravity $\left(9.81 \mathrm{~m} / \mathrm{s}^{2}\right)$.

The right selection of mass $m$ is critical in the manufacturing process. If this mass is too high the fiber may break. Conversely, if it is not enough the fiber will bend instead of coil. In the case of this work, two different threads of diameter $0.2 \mathrm{~mm}$ and $0.7 \mathrm{~mm}$ where used. Using $17 \mathrm{MPa}$ as the value for $T$, out of [8], weights of 54 and 667 grams where obtained using Equation 1. With experimental validations, it can be assert that this values are the lower limit for avoiding bending, there is a margin up to the upper limit, where the fibers brake.

As summary of the fabrication process of one muscle fiber, first step is to cut a desired length of the Nylon thread. Then, the thread is placed on a test-bench, and coiled with the recommended weight for applying tension. After a time, the thread will start coiling forming loops. When all the loops are formed, the coiling process must be stopped and the annealing can start. The first annealing can be done with any load, as its function is to eliminate residual tensions. Later on, a second annealing with more weight must be performed to create space between loops and allow compression. Finally, after this, the muscle fiber will be ready to use.

The direction of formation of the loops can be the same (homochiral) or the opposite (heterochiral). Homochiral refers to muscles fibers in which the direction of the coils are in the same spin than the internal twist deformation. 
In the heterochiral case, the spin of the coils occurs in the opposite direction of the internal torsion of the nylon. This difference makes that, when heated, the first type tends to compress and the second one tends to expand. Most of the works $[20,18,14]$ focus on the use of homochiral muscles. On the other hand, heterochiral fibers are as best defined in the first paper published, [8], this expansive effect may be of interest for other specific applications.

The manufacturing process of heterochiral fibers is more complex due to the need of inverting the torsion in the coiling phase. This is typically done by manually coiling the muscle around a rod. Conversely, homochiral fibers can be created with and without a rod, because the coiling phase appears spontaneously when the nylon collapses (self-coiled fibers).

Using a rod for the manufacturing process has advantages and disadvantages. On one hand, manually rod-coiled muscles have a higher internal diameter (equal to the diameter of the rod). Enlarging this diameter allows the muscles to compress or expand more than the self-coiled ones (up to $50 \%$ compared with the 10-20\% obtained by self-coiled). Also, this method allows to separate the loops. This fact is important, because it enables the muscle to compress even if it is not stressed (preloaded), due to the existing space between the loops. This behaviour is related with the elastic properties of the Nylon, such as elastic range and limit [6]. Conversely, such muscle fibers have less lifting force, being it inversely proportional to the diameter of the loops. Self-coiled muscle fibers have the minimum loop diameter and so the maximum force [8]. The problem with self-coiled fibers is that the loops do not have inter-distance, so that to be able to compress them, they should be previously stressed.

However, the experimentation made in this work has proven that self-coiled muscles fibers modify their length after a few annealing cycles, increasing their length notoriously compared with its initial length just after the coiling. This increment depends on the hanging weight. It means, that the muscle adjusts its length to the force applied on it when annealed. As a consequence, spaces between the loops of the spring are generated. Therefore, the muscle fiber will have an initial length between there loops, allowing it to contract even if not stressed. Only a minimum initial force must be applied to stretch the muscle fiber completely.

Nevertheless, the annealed state is reversible, i.e., if the muscle tensor is stored without any external force applied on it, it will eventually recover the original length, it had before the annealing, due to the shape memory property of the nylon. If that happens, the muscle can be retrained to recover the previous properties, using the same weight.

In order to obtain the model of the system, we have analyzed the behavior of the TCP muscles in two scenarios. At first, the muscles have been tested in a vertical test-bench, loaded with different weights and actuated with an electrical source. Then, a second set up has been prepared using a muscle fiber stressed by a spring. The idea behind this setup is to build a bio-inspired dual muscle system with antagonist actuator. 


\subsection{Test bench with free weight}

Figure 1 shows the basic set up. The muscle is held on one side, and a load is placed on the other side.

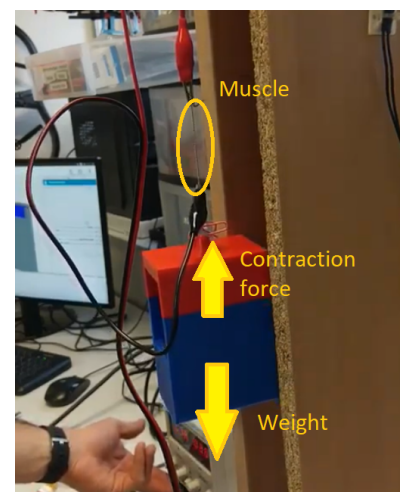

Fig. 1. Existing forces in the free-weight test-bench

The muscle behaves like a spring with no heating actuation. Thus, applying equilibrium of forces Equation 2 is obtained.

$$
K \cdot \Delta l=F_{\text {ext }}=m \cdot g
$$

$K$ means the static factor characteristic of the muscle, $\Delta l$ is the increment of length of the muscle due to the load, $m$ is the mass of the load and $g$ is the gravity force. If the system is analyzed statically, it can be observed that at each instant, the equilibrium of forces is:

$$
\begin{aligned}
& K \cdot \Delta l+F_{\text {muscle }}=m \cdot g \\
& F_{\text {muscle }}=m \cdot g-K \cdot \Delta l
\end{aligned}
$$

From the static point of view, the muscle acts with a force that is equal to the strength lost by the internal spring force but with the opposite direction. When heated, the Nylon tries to recover the untwisted state (shape memory), producing a force in the longitudinal direction and compressing the coils. This phenomena is modeled as a force $F_{\text {muscle }}$ that compensates the decrease of $\Delta l$ of the spring composed by muscle's coils.

Figure 2 shows the evaluation of the muscle against different loads given fixed energy from the power source.

On the left image, it can be observed the length of the fiber against different weights in three different situations. $l_{0}$ refers to the initial static length of the unannealed fiber. Variable $l_{\text {elon }}$ refers to the length of the fiber after the annealing. The last line, $l_{\text {compr }}$, is the maximum compression achieved with the fiber against 

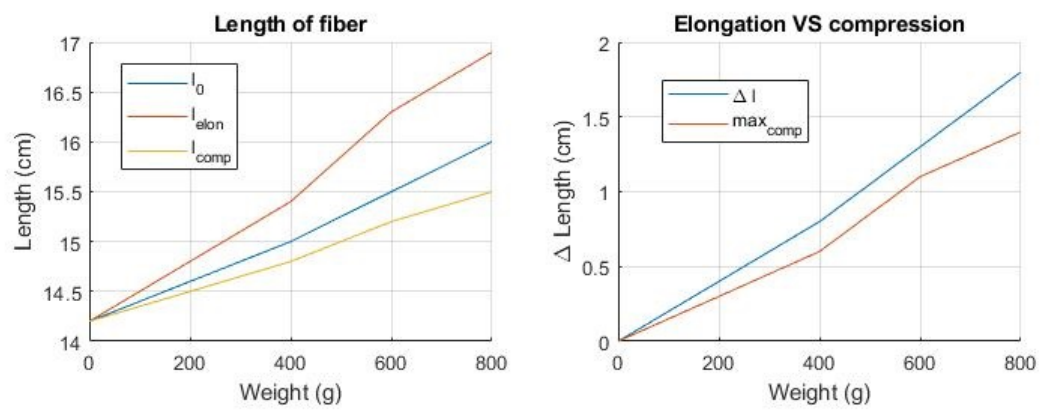

Fig. 2. Evaluation of muscle fiber against different fixed loads.

the different loads. Right figure shows the absolute elongation and compression. This figure shows that the fiber recovers almost all the elongation when actuated.

When the muscle is heated, the force of the muscle grows and the length of the muscle decreases, maintaining the balance between forces. This assertion leads to the following conclusions:

- muscles need to have internal space to be able to compress and exert forces longitudinally.

- when fully compressed, the force is still present but can not be used to actuate.

- if the muscle is still actuated after reaching the maximum compression, it starts bending, trying to recover the untwisted shape.

- if this last state is reached, the muscle fiber will be near to collapse and break.

\subsection{Bio-inspired Muscle-Antagonist configuration}

From the previous experiments, we determined that the muscles can at least generate forces to lift fixed external forces. However, in this new set up, the muscle will stretch a spring, which means that the external force will increase as it self-compress. The reason for this new set up is to be able to reproduce a muscle antagonist pair, typical in animals. Consequently, when a muscle actuates, the second one, or antagonist, acts as a passive spring.

Figure 3 shows the proposed configuration. Equation 5 shows the proposed model for the equilibrium of forces for this set-up.

$$
K_{1} \Delta l_{1}(t)=K_{2} \Delta l_{2}(t)+F_{\text {muscle }}(T, t)
$$

Where $F_{\text {muscle }}$ is dependant of the temperature. Temperature is, on the other hand dependant of the time, due to the fact that it increase with it when a tension is applied thanks to the Joule effect. $l_{1}$ and $l_{2}$ modules are the same, when one of the terms decrease the other increases. $K_{1}$ is the stiffness coefficient 

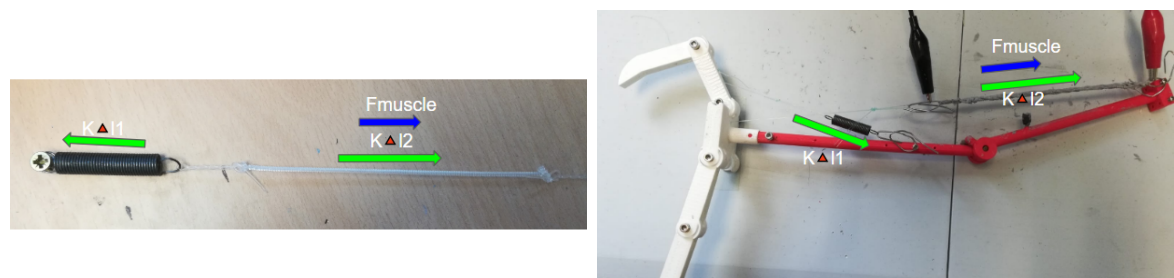

Fig. 3. On the right, spring-muscle configuration. On the left, early version of the claw with one finger actuated by a muscle fiber and a passive spring.

of the spring and $K_{2}$ is the stiffness coefficient of the muscle fiber as seen in Equation 2, being both of them constant. However, the increase of the $F_{\text {muscle }}$ is able to compensate that decrease and increase and get to a new equilibrium. Doing regression to the experimental data, a quadratic regression is obtained for the model of the muscle fiber behaviour.

Figure 4 shows the compression of the muscle in the presented configuration. At first, the experiments show that the muscle overpass the force of the fixed spring, which is a necessary condition to validate the configuration.

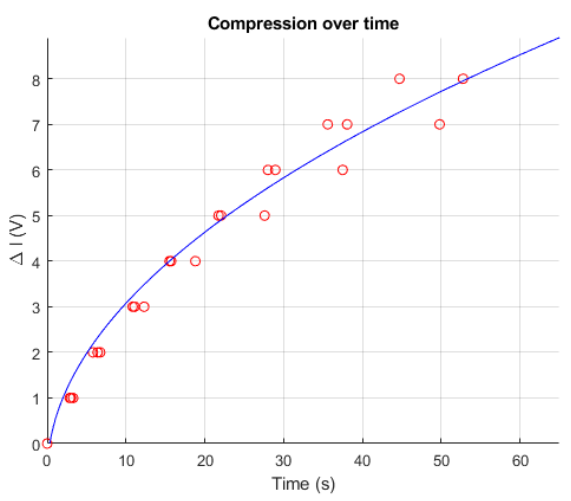

Fig. 4. Muscle fiber stressed by a spring, the force generated are shown

The muscle starts compressing as in the fixed-load experiments. However, due to the increment of $\Delta l$ the force of the passive spring increases, opposing the muscle. As more the muscle compress, more is the force that the spring exert. Thus larger is the force that the muscle need to generate per unit of compression, or equivalently, smaller is the compression per unit of force. Figure 4, show the compression of the muscle against the spring over time. Contrary to Figure 2 the trend is not linear.

According to that, the chosen model is an square root equation. For this model it is assumed that the muscle is heated by the Joule effect by applying a 
fixed voltage. Precisely, these experiments were carried out with 5 Volts,consuming around 10 Watts of power. Additionally, the environment conditions remain over the experiments. The energy efficiency of the TCP is low. However, due to the reduce of weight by using these tensors instead of servos and the relatively low consumption, compared with the one of the brushless motors, the UAV autonomy is not reduced.

$$
\Delta l=a+b \cdot \sqrt{x}
$$

\section{Hybrid Self-coiled Muscles (HSM)}

The design of the muscle used in this work tries to get the best performance and reliability using the existing technologies. For that purpose, a combination of Nylon wires is used. A non-conductive fishing line nylon thread of $0.7 \mathrm{~mm}$ diameter is coiled and used as core fiber for the muscle. This fiber can lift a large weight without breaking, but it does not have any conductive material for self-heating it. To overcome this issue, several muscle fibers of Shieldex 235/36 4-ply silver covered nylon wire are coiled with the previous fishing line wire. This muscle fibers, made of the Shieldex material, can lift up to 200 grams each, and they can be actuated electrically. Figure 5 shows the resulting muscle hybrid after the first annealing phase.

Particularly, for the experiments conducted in this article, five conductive fibers were coiled around the non conductive nylon thread. This muscle composition has been characterized for the model presented in Equation 6 having the following parameters $(a, b)=$

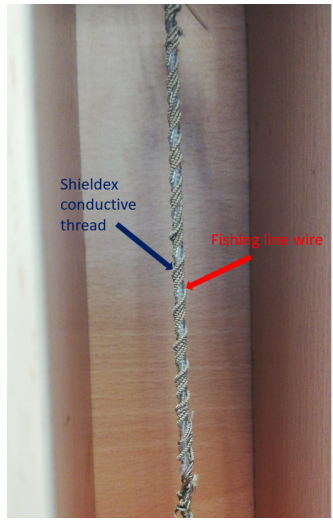

Fig. 5. An hybrid 6-ply muscle out of one fishing line $(0.7 \mathrm{~mm})$ muscle fiber and 5 Shieldex conductive $(0.2 \mathrm{~mm})$ silver threads $(-0.6815,1.188)$ with a confidence bound of $95 \%$.

The resulting muscle, is capable of lifting up to 1 kilogram. With this solution, the system is capable of put up with real objects, lifting them without opening the limbs with the force generated by the weight. This is an important fact, because state of art muscles do have large limitations in terms of payload capabilities [13].

\section{Application in Aerial Manipulation. Ultra Lightweight Claw}

In this section, a preliminary version of an eagle-based claw with three fingers to be actuated with muscles fibers for ornithopters is presented. Taking inspiration in birds configuration, the muscles are placed on the legs as straight as possible, giving the muscle freedom to move and avoiding friction as much as possible. 
At the tip of the muscles, normal fishing lines thread is placed. These act as tendons to transmit the force needed to the fingers. The idea of using fishing line as tendons is due to the property of nylon of not stretching and being resistant. All the designs are printed using additive manufacturing using Polylactic acid (PLA) and Filaflex to create flexible joints. Figure 6 shows the design of the claw. The distributions of the fingers mimics real eagle fingers.
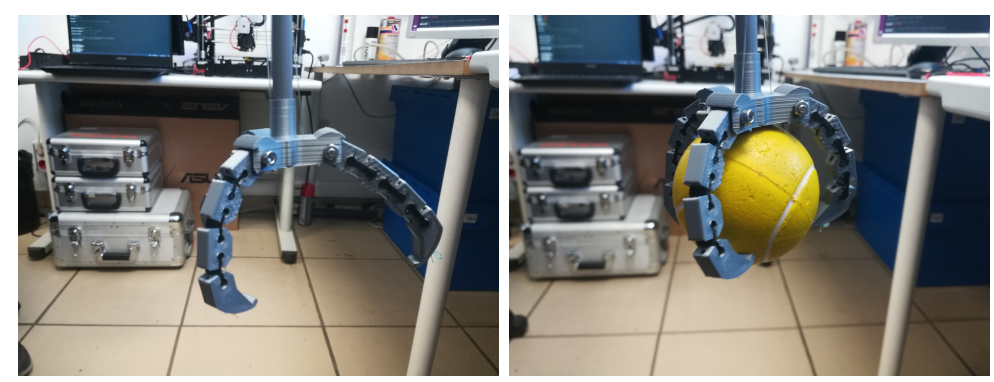

Fig. 6. Grasp of a plastic ball by the three finger of the claw, it can be observed that the claw can put up with the weight even looking downwards

For actuating all the phalanges with just one muscle fiber, the joints are made of Filaflex which, when not actuated, act as a spring, returning to their rest position. The resting state is the extended finger. When actuated, the Filaflex will apply some resistance to the movement, that will be enough for keeping the muscle stretched.

Also, this design provides smoothness to the movement of the claw, fact that we did not achieve using common axial joints. The fingers act as one articulation, composed by many, with Filaflex "springs", connected articulations.

\section{Conclusions}

This paper has presented the foundations for working with Twisted and Coiled Polymers for aerial manipulators. At first, the manufacturing process has been wrapped up. This is one critical step in this new trend of AMs. Due to the absence of existing manufacturing is essential to establish standard procedures for the developers.

Then, a simple but yet effective model has been proposed to analyze the behaviour of the proposed muscles. These models have been validated experimentally in different experiments.

It is important to remark that the use of these actuators may greatly impact the field of aerial manipulators, providing ultra-lightweight actuators that overtake the capabilities of existing servomotors. Moreover, in the field rising field of ornithopters, which payload capabilities are extremely limited. Embedding this muscles will lead to a new generation of aerial robots. 


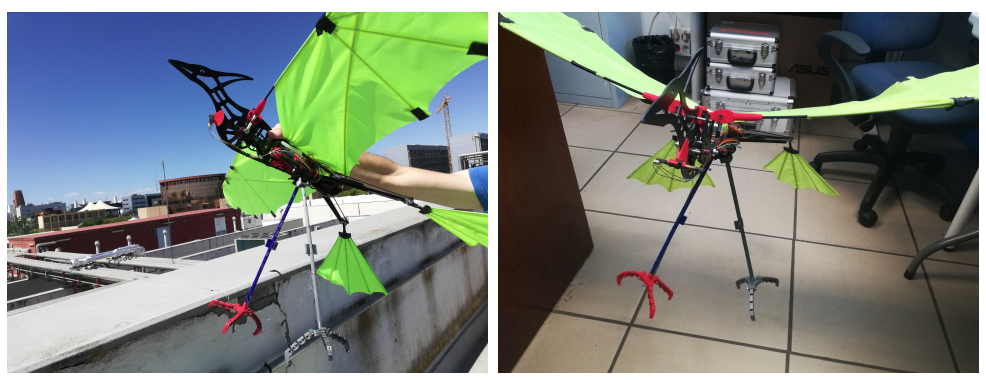

Fig. 7. Two claws on an ornithopter used for the project GRIFFIN an european ERC advanced grant

Nevertheless, there is still a lot of intense research that needs to be developed in this field. Next steps will focus in developing a control system for this actuators. Also, exploiting the combination of TCP muscles with other bio-inspired/soft actuators may lead develop more efficient solutions in aerial robotics and replace traditional actuators.

\section{Acknowledgments}

We thank Robotics, Vision and Control Group for supporting us during this work. This work has been developed under the framework of the project GRIFFIN (General compliant aerial Robotic manipulation system Integrating Fixed and Flapping wings to INcrease range and safety) SI-1867/23/2018 ERC-ADG - Advanced Grant EU-funded project.

\section{References}

1. Arakawa, T., Takagi, K., Tahara, K., Asaka, K.: Position control of fishing line artificial muscles (coiled polymer actuators) from nylon thread. In: Electroactive Polymer Actuators and Devices (EAPAD) 2016. vol. 9798, p. 97982W. International Society for Optics and Photonics (2016)

2. Arrue, M.P.J.P.R.S.B.C., Ollero, A.: Hecatonquiros: Open-source hardware for aerial manipulation applications. International Journal of Advanced Robotic Systems 16(3) (2019)

3. Arrue, P.R.A.G.F.G.B., Ollero, A.: Autonomous landing on pipes using soft gripper for inspection and maintenance in outdoor environments. In: 2019 IEEE/RSJ International Conference on Intelligent Robots and Systems (IROS) (Nov 2019)

4. Bartelds, T., Capra, A., Hamaza, S., Stramigioli, S., Fumagalli, M.: Compliant aerial manipulators: Toward a new generation of aerial robotic workers. IEEE Robotics and Automation Letters 1(1), 477-483 (Jan 2016)

5. Bellicoso, C.D., Buonocore, L.R., Lippiello, V., Siciliano, B.: Design, modeling and control of a 5-dof light-weight robot arm for aerial manipulation. In: 2015 23rd Mediterranean Conference on Control and Automation (MED). pp. 853-858. IEEE (2015) 
6. Cherubini, A., Moretti, G., Vertechy, R., Fontana, M.: Experimental characterization of thermally-activated artificial muscles based on coiled nylon fishing lines. Aip Advances 5(6), 067158 (2015)

7. DING, X., GUO, P., XU, K., YU, Y.: A review of aerial manipulation of small-scale rotorcraft unmanned robotic systems. Chinese Journal of Aeronautics 32(1), $200-$ 214 (2019), http://www.sciencedirect.com/science/article/pii/S1000936118301894

8. Haines, C.S., Lima, M.D., Li, N., Spinks, G.M., Foroughi, J., Madden, J.D., Kim, S.H., Fang, S., de Andrade, M.J., Göktepe, F., et al.: Artificial muscles from fishing line and sewing thread. science 343(6173), 868-872 (2014)

9. Ignasov, J., Kapilavai, A., Filonenko, K., Larsen, J.C., Baird, E., Hallam, J., Büsse, S., Kovalev, A., Gorb, S.N., Duggen, L., et al.: Bio-inspired design and movement generation of dung beetle-like legs. Artificial Life and Robotics 23(4), 555-563 (2018)

10. Ollero, A., Heredia, G., Franchi, A., Antonelli, G., Kondak, K., Sanfeliu, A., Viguria, A., Martinez-De Dios, J.R., Pierri, F., Cortés, J., et al.: The aeroarms project: Aerial robots with advanced manipulation capabilities for inspection and maintenance. IEEE Robotics and Automation Magazine (2018)

11. Oszwald, F., Wedler, A., Schiele, A.: Development of a bioinspired robotic insect leg (11 2004)

12. Ruggiero, F., Lippiello, V., Ollero, A.: Aerial manipulation: A literature review. IEEE Robotics and Automation Letters 3(3), 1957-1964 (2018)

13. Saharan, L., de Andrade, M.J., Saleem, W., Baughman, R.H., Tadesse, Y.: igrab: hand orthosis powered by twisted and coiled polymer muscles 26(10), 105048 (2017), https://app.dimensions.ai/details/publication/pub.1091450247andhttp: //iopscience.iop.org/article/10.1088/1361-665x/aa8929/ampdf, exported from https://app.dimensions.ai on 2019/05/28

14. Semochkin, A.N.: A device for producing artificial muscles from nylon fishing line with a heater wire. In: 2016 IEEE International Symposium on Assembly and Manufacturing (ISAM). pp. 26-30. IEEE (2016)

15. Suarez, A., Heredia, G., Ollero, A.: Lightweight compliant arm for aerial manipulation. In: 2015 IEEE/RSJ International Conference on Intelligent Robots and Systems (IROS). pp. 1627-1632 (Sep 2015)

16. Suarez, A., Soria, P.R., Heredia, G., Arrue, B.C., Ollero, A.: Anthropomorphic, compliant and lightweight dual arm system for aerial manipulation. In: 2017 IEEE/RSJ International Conference on Intelligent Robots and Systems (IROS). pp. 992-997. IEEE (2017)

17. Sun, B., Jing, X.: A tracked robot with novel bio-inspired passive legs. Robotics and biomimetics 4(1), 18 (2017)

18. Wu, L., de Andrade, M.J., Saharan, L.K., Rome, R.S., Baughman, R.H., Tadesse, Y.: Compact and low-cost humanoid hand powered by nylon artificial muscles. Bioinspiration \& biomimetics 12(2), 026004 (2017)

19. Wu, L., Karami, F., Hamidi, A., Tadesse, Y.: Biorobotic systems design and development using tcp muscles. In: Electroactive Polymer Actuators and Devices (EAPAD) XX. vol. 10594, p. 1059417. International Society for Optics and Photonics (2018)

20. Yip, M.C., Niemeyer, G.: High-performance robotic muscles from conductive nylon sewing thread. In: 2015 IEEE International Conference on Robotics and Automation (ICRA). pp. 2313-2318. IEEE (2015) 